\title{
Transient MHD Double-Diffusive Natural Convection over a Vertical Surface Embedded in a Non-Darcy Porous Medium
}

\author{
Mohammed Q. Al-Odat, ${ }^{1}$ Tariq A. Al-Azab, ${ }^{2}$ \\ M. Al-Hasan, ${ }^{3}$ and B. A. Shannak ${ }^{1}$ \\ ${ }^{1}$ Mechanical Engineering Department, Al-Huson University College, Al-Balqa Applied University, \\ P.O. Box 21510, Irbid, Jordan \\ ${ }^{2}$ Mechanical Engineering Department, Al-Salt Community College, Al-Balqa Applied University, \\ Al-Salt 19117, Jordan \\ ${ }^{3}$ Mechanical Engineering Department, Faculty of Engineering and Technology, \\ Al-Balqa Applied University, P.O. Box 340558, Amman 11134, Jordan
}

Correspondence should be addressed to Mohammed Q. Al-Odat, m_alodat@yahoo.com

Received 18 January 2009; Revised 4 August 2009; Accepted 12 November 2009

Recommended by Kumbakonam R. Rajagopal

The problem of transient, laminar, MHD double-diffusive free convection over a permeable vertical plate embedded in Darcy and non-Darcy porous medium is numerically investigated. Nonsimilarity solutions are obtained for constant wall temperature and concentration with a specified power law of mass flux parameter. The effects of the magnetic parameter, the inertial coefficient, Lewis number, the buoyancy ratio, and the lateral mass flux on heat and mass transfer coefficients are presented and discussed.

Copyright (C) 2009 Mohammed Q. Al-Odat et al. This is an open access article distributed under the Creative Commons Attribution License, which permits unrestricted use, distribution, and reproduction in any medium, provided the original work is properly cited.

\section{Introduction}

Recently, heat and mass transfer from different geometries embedded in fluid saturated porous medium has been studied extensively. This is due to the fact that these flows have many engineering and geophysical applications which include geothermal resources, building insulation, oil extraction, underground disposal of nuclear waste, heat salt leaching in soils, and many more. Various researchers [1-3] have studied heat and mass transfer by free convection in porous medium under boundary layer approximation. It is worth mentioning that all these studies were focused on steady state conditions and based on Darcy model. In contrast, transient convective flow problems have not received as much attention. 
This is because that the transient heat transfer is usually difficult to solve either analytically or numerically. In fact there is no actual flow situation, which does not involve unsteadiness, and examples of transient convective flows are numerous, for example, cooling of electronic devices in which the heat generation is not constant but time varying. Darcy model is valid when the Reynolds number based on the pore size is less than unity. For fluids of high velocity and/or porous material of large pore radius, the Darcy model is inadequate since it neglects the porous medium inertia effect, which becomes significant. Inertial effects are taken into consideration in Forchheimer flow model, which is a modification of the original Darcy law by adding the quadratic inertial term [4-6].

There has been a renewed interest in MHD flow and heat transfer in porous and clear domains due to the important effect of magnetic field on the performance of many systems using electrically conducting fluid such as MHD power generators and the cooling of nuclear reactors. In a review article [7], Ram presented an account of several steady MHD heat and mass transfer problems. Chamkha [8] considered the steady MHD free convection from vertical plate in a thermally stratified porous medium with Hall effect.

The aim of this work is to investigate transient MHD double-diffusive of an electrically-conducting fluid by free convection over a flat plate embedded in Darcy and non-Darcy porous medium in the presence of surface suction or blowing and magnetic field effects.

\section{Mathematical Analysis}

Consider the unsteady non-Darcy MHD free double-diffusive convection flow of an electrically conducting fluid over an isothermal vertical porous plate embedded in a porous medium with suction or injection. Initially the wall is at constant temperature $T_{\infty}$ and concentration $C_{\infty}$, respectively. At $t=0$, the wall temperature and concentration are suddenly raised to $T_{w}$ and $C_{\infty}$, higher than the ambient values $T_{\infty}$ and $C_{\infty}$, respectively and they maintained at these values for $t>0$. A uniform magnetic field is applied normal to the plate. It is assumed that, the fluid properties are constant except the influence of density variation with temperature which is considered only in the body force term, both fluid and solid matrix are in local thermal equilibrium; the permeability porous medium is constant, viscous dissipation; Joule heating and thermal dispersion effects are negligible. Under these assumptions along with Boussinesq approximation, the governing equations are given by

$$
\begin{gathered}
\frac{\partial u}{\partial x}+\frac{\partial v}{\partial y}=0 \\
\left(1+\frac{\sigma B_{0}^{2} K}{\rho v}\right) \frac{\partial u}{\partial y}+\frac{C \sqrt{K}}{v} \frac{\partial u^{2}}{\partial y}=\left(\frac{K g \beta_{T}}{v}\right) \frac{\partial T}{\partial y}+\left(\frac{K g \beta_{C}}{v}\right) \frac{\partial C}{\partial y} \\
\sigma \frac{\partial T}{\partial t}+u \frac{\partial T}{\partial x}+v \frac{\partial T}{\partial y}=\alpha_{e} \frac{\partial^{2} T}{\partial y^{2}} \\
\varphi \frac{\partial C}{\partial t}+u \frac{\partial C}{\partial x}+v \frac{\partial C}{\partial y}=D_{m} \frac{\partial^{2} C}{\partial y^{2}}
\end{gathered}
$$


The physical problem suggests the following initial and boundary conditions:

$$
\begin{gathered}
u(x, y, 0)=0, \quad v(x, y, 0)=0, \quad T(x, y, 0)=T_{\infty}, \quad C(x, y, 0)=C_{\infty}, \\
v(x, 0, t)=v_{w}(x)=\frac{A}{\sqrt{x}}, \quad T(x, 0, t)=T_{w}, \quad C(x, 0, t)=C_{w} \\
u(x, \infty, t)=0, \quad T(x, \infty, t)=T_{\infty}, \quad C(x, \infty, t)=C_{\infty}
\end{gathered}
$$

where $(x, y)$ are the dimensional distance along and normal to the plate, respectively, $(u, v)$ and are the averaged velocity components along the $x$ and $y$, directions respectively, $t$ is the time, $\sigma$ is the heat capacity ratio of the porous media, $\sigma=\varphi+(1-\varphi)(\rho c)_{s} /(\rho c)_{s^{\prime}} T$ is the temperature, $C$ is the concentration, $\beta_{T}$ and $\beta_{c}$ are the thermal expansion coefficient and the concentration expansion coefficient respectively, $v$ is the effective kinematic viscosity, $\alpha_{e}$ is the effective thermal diffusivity, $F$ is the dimensional inertial coefficient, $K$ is the permeability of the medium, respectively, $V_{w}$ is the suction $(>0)$ or injection $(<0)$ velocity, $\varphi$ is the porosity of the porous medium, and $D_{m}$ is the mass diffusion coefficient.

Introducing the following dimensionless variables and parameters:

$$
\begin{gathered}
\eta=\frac{y}{x} \sqrt{\operatorname{Ra}_{x}}, \quad \psi=\alpha \sqrt{\operatorname{Ra}_{x}} f(\eta, \tau), \quad \tau=\frac{\alpha \mathrm{Ra}_{x} t}{\sigma x^{2}}, \quad u=\frac{\partial \psi}{\partial y}, \quad v=-\frac{\partial \psi}{\partial x}, \\
\theta=\frac{T-T_{\infty}}{T_{W}-T_{\infty}}, \quad \phi=\frac{C-C_{\infty}}{C_{W}-C_{\infty}}, \quad \mathrm{Le}=\frac{\alpha}{D}, \quad \mathrm{Ha}=\sqrt{M}=\sqrt{\frac{\sigma B_{0}^{2} K}{\rho v}}, \quad N=\frac{\beta_{C}\left(C_{W}-C_{\infty}\right)}{\beta_{T}\left(T_{W}-T_{\infty}\right)}, \\
\operatorname{Ra}_{x}=\frac{K g \beta_{T}\left(T_{W}-T_{\infty}\right) x}{\alpha v}, \quad \Gamma=\frac{F \sqrt{K} K g \beta_{T}\left(T_{W}-T_{\infty}\right)}{v^{2}}, \quad f_{w}=-\frac{2 x v_{w}(x)}{\alpha \sqrt{\mathrm{Ra}_{x}}},
\end{gathered}
$$

where $\psi$ is the stream function, $\tau$ is the dimensionless time, $\Gamma$ is the Forchheimer number, $\mathrm{Ha}$ is the Hartmann number, $M$ is the magnetic parameter, Le is the Lewis number, $\mathrm{Ra}_{x}$ is the modified Rayleigh number, $f_{w}$ is the mass flux parameter. $f_{w}$ is varied from -1 to 1 . It is obvious that $f_{w}=0$ corresponds to impermeable wall, $f_{w}>0$ corresponds to suction and $f_{w}<0$ corresponds to injection.

Using the above similarity transformation the governing equations are reduced to

$$
\begin{gathered}
\left(1+\mathrm{Ha}^{2}\right) f^{\prime \prime}+2 \Gamma f^{\prime \prime} f^{\prime}=\theta^{\prime}+N \phi^{\prime}, \\
\theta^{\prime \prime}=-\frac{1}{2} f \theta^{\prime}+\frac{\partial \theta}{\partial \tau}+\tau\left[f^{\prime} \frac{\partial \theta}{\partial \tau}-\theta^{\prime} \frac{\partial f}{\partial \tau}\right], \\
\phi^{\prime \prime}=-\frac{1}{2} \operatorname{Le} f \phi^{\prime}+\frac{\sigma}{\varphi} \frac{\partial \phi}{\partial \tau}+\tau\left[f^{\prime} \frac{\partial \phi}{\partial \tau}-\phi^{\prime} \frac{\partial f}{\partial \tau}\right],
\end{gathered}
$$


and the initial and boundary conditions are transformed to

$$
\begin{aligned}
& \quad f(\eta, 0)=\theta(\eta, 0)=0.0, \\
& \text { at } \eta=0, \quad f=f_{w}, \quad \theta=1, \quad \phi=1, \\
& \text { as } \eta \longrightarrow \infty, \quad f^{\prime}=0, \quad \theta=0, \quad \phi=0 .
\end{aligned}
$$

The physical quantities of fundamental interest of heat and mass transfer study are the heat and mass coefficients in terms of Nusselt and Sherwood numbers, respectively. The dimensionless heat and mass transfer coefficients can be expressed as

$$
\frac{\mathrm{Nu}}{\sqrt{\mathrm{Ra}_{x}}}=-\theta^{\prime}(0), \quad \frac{\mathrm{Sh}}{\sqrt{\mathrm{Ra}_{x}}}=-\phi^{\prime}(0) .
$$

\section{Results and Discussion}

The resulting partial differential equations together with their boundary conditions have been solved numerically using an implicit finite difference technique. For the sake of brevity, the details of the solution procedure are not presented here. The results reported in this paper were validated by comparing them with those in the previously published paper. Our results show an excellent a greement with the steady state results of [9].

A parametric study is carried out to investigate the effects of all involved parameters on the transient velocity, temperature and concentration profiles as well as the transient local Nusselt and the local Sherwood numbers.

The effects of magnetic parameter on the transient velocity profiles, temperature distribution, and concentration distribution in the non-Darcy flow region are shown in Figures 1 and 2. It is clear that hydrodynamics, thermal and concentration boundary layers thicken gradually with time. The presence of the magnetic field produces a resistive force that decelerates the fluid flow in the porous medium and increases the thermal and concentration boundary layer thicknesses.

Figure 3 illustrates the influence of the mass flux parameter on the transient velocity profiles. It can be seen that the velocity increases as the mass flux parameter passes from suction to injection region.

The effect of the buoyancy ratio on the local Nusselt number with and without magnetic field effect is displayed in Figure 4. This figure depicts the enhanced heat transfer results with the buoyancy ratio. It is clearly seen that the presence of magnetic field reduces the heat transfer coefficient. Furthermore, the effect of lateral mass flux is predominating for higher values of buoyancy ratio.

The influences of lateral mass flux on the local Nusselt number and the local Sherwood number with and without magnetic field effect and at different values of Lewis number are displayed in Figures 5 and 6, respectively. The increase in the Lewis number reduces heat transfer rate, where as it increases the mass transfer rate. The magnetic field effect reduces both the heat and mass transfer coefficients. It is clear that the suction increases both the heat and mass transfer rates. 


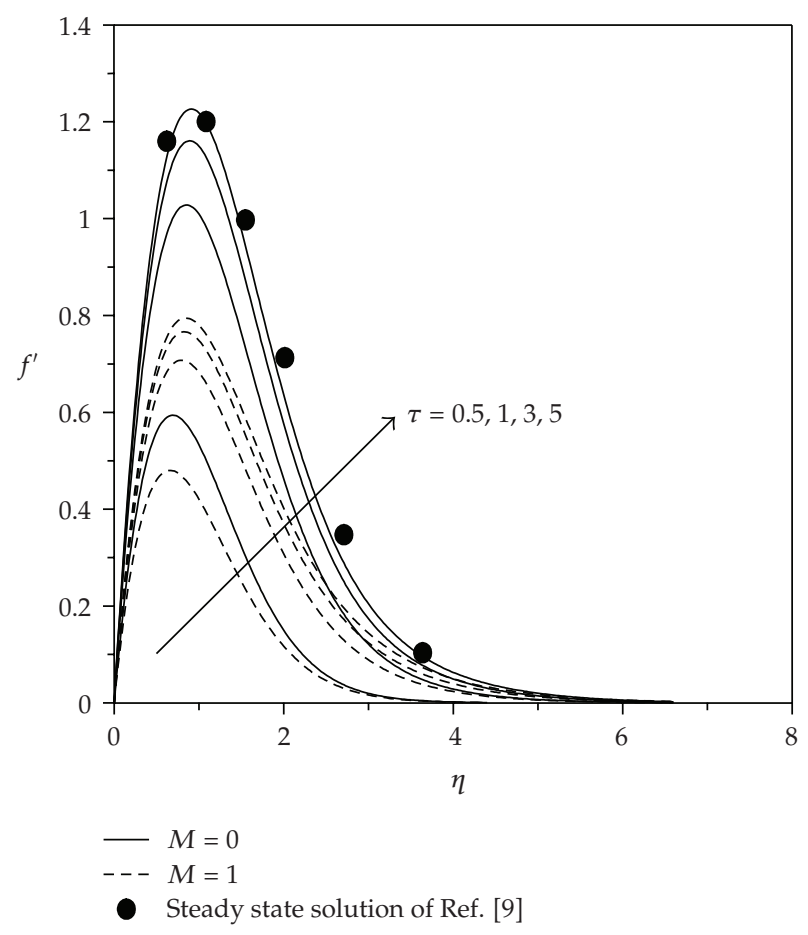

Figure 1: The effect of magnetic parameter on the transient dimensionless velocity component profiles $\left(N=1, \mathrm{Le}=2, \Gamma=1\right.$, and $\left.f_{w}=0\right)$.

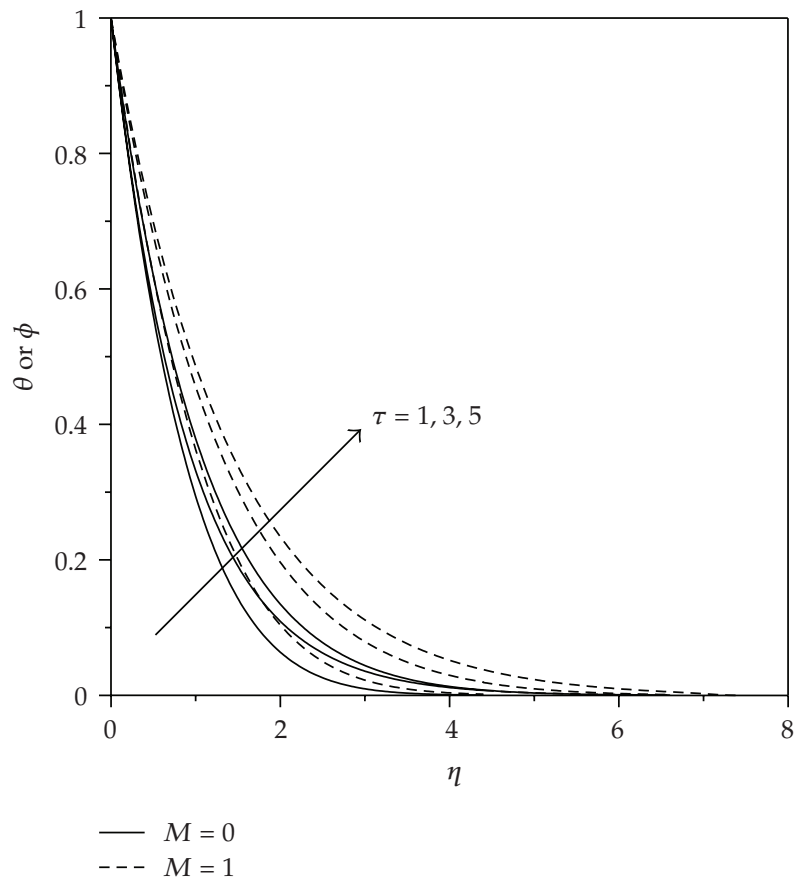

Figure 2: The effect of magnetic parameter on the transient dimensionless temperature distribution $(N=1$, Le $=2, \Gamma=1$, and $\left.f_{w}=0\right)$. 


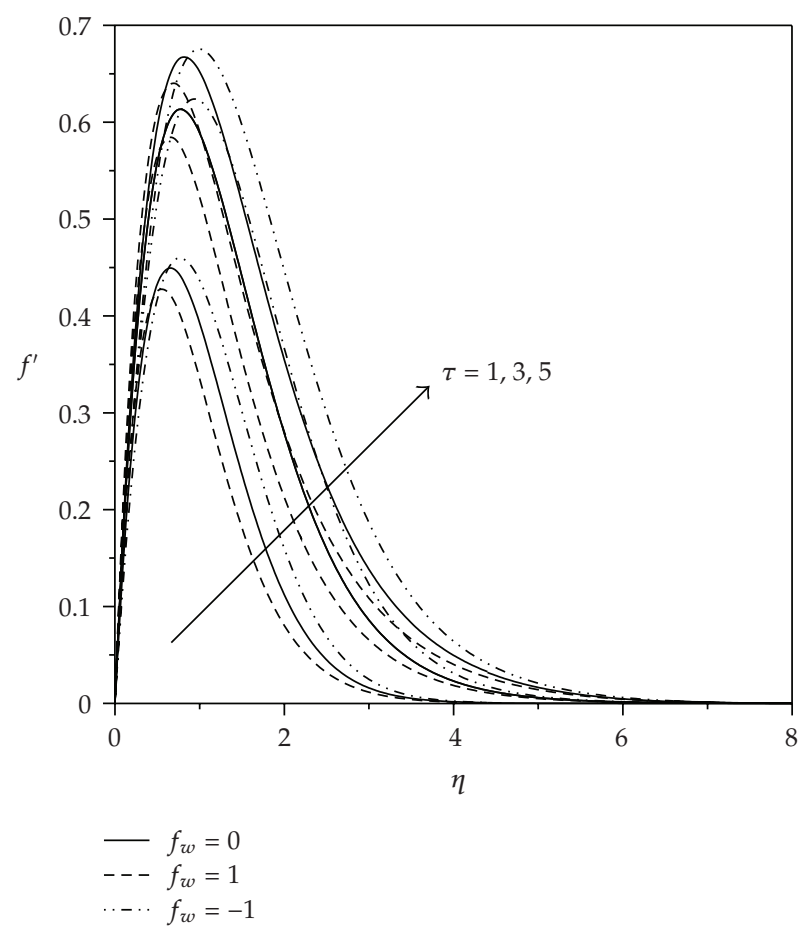

Figure 3: The effect of magnetic parameter on the transient dimensionless temperature distribution, for suction, injection, and impermeable wall cases $(N=1, \operatorname{Le}=2$, and $\Gamma=1)$.

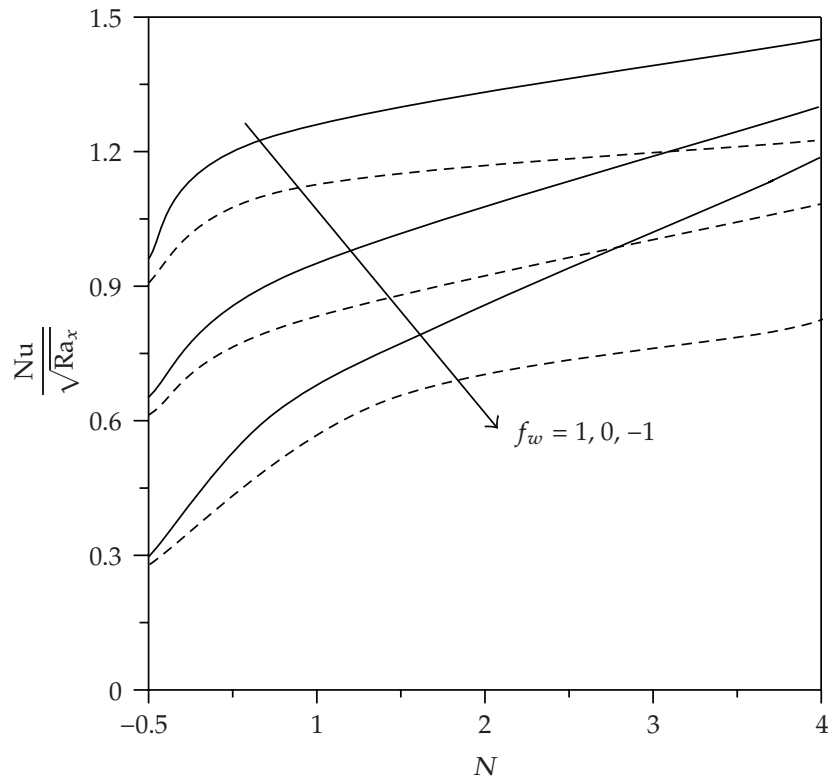

$$
\begin{aligned}
-M & =0 \\
--- & M=1
\end{aligned}
$$

Figure 4: The effect of buoyancy ratio on Nusselt number with and without magnetic field effect, for suction, injection, and impermeable wall cases $(N=1, \operatorname{Le}=2, \tau=1$, and $\Gamma=1)$. 


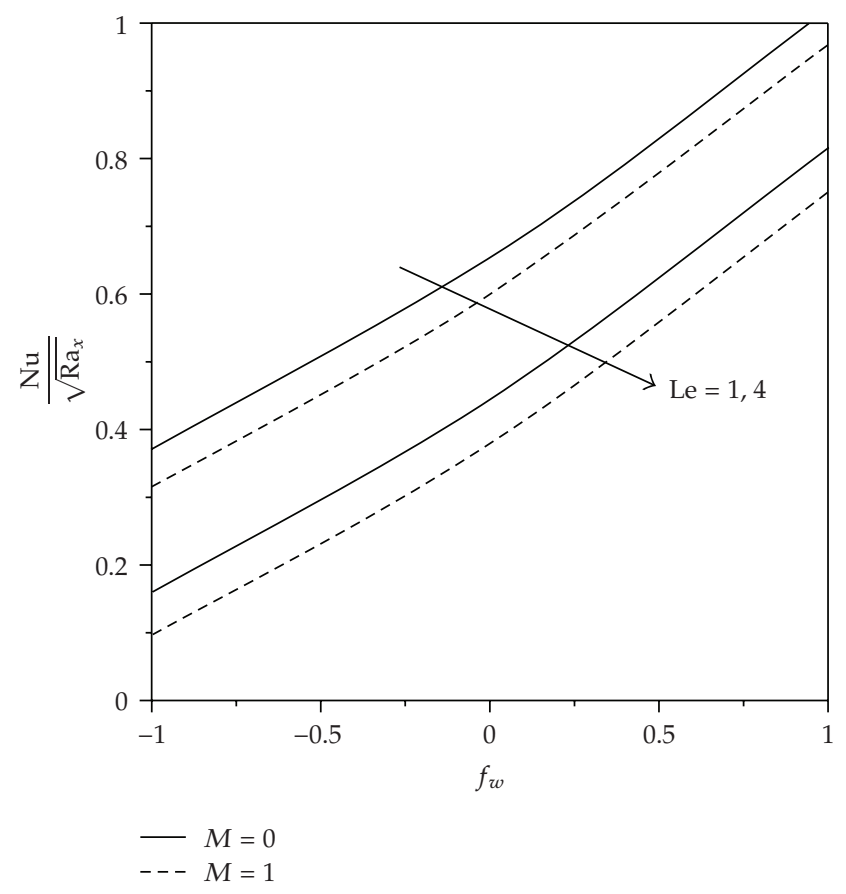

Figure 5: The effect of mass flux parameter on Nusselt number with and without magnetic field effect at different values of Lewis number $(N=1, \tau=1$, and $\Gamma=1)$.

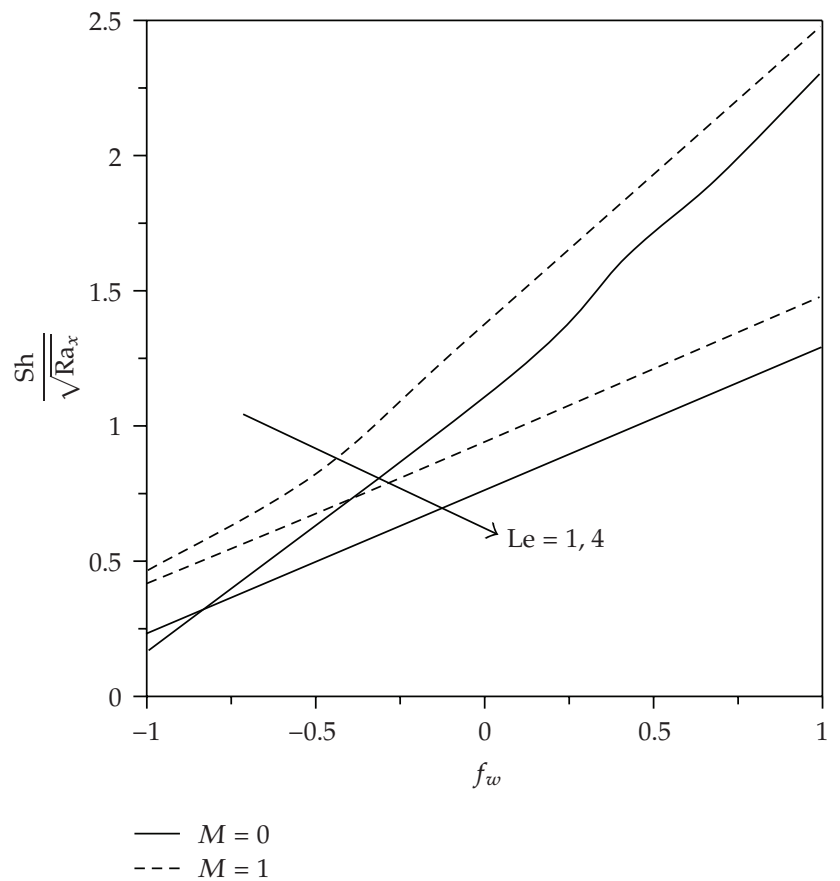

Figure 6: The effect of mass flux parameter on Sherwood number with and without magnetic field effect at different values of Lewis number $(N=1, \tau=1$, and $\Gamma=1)$. 


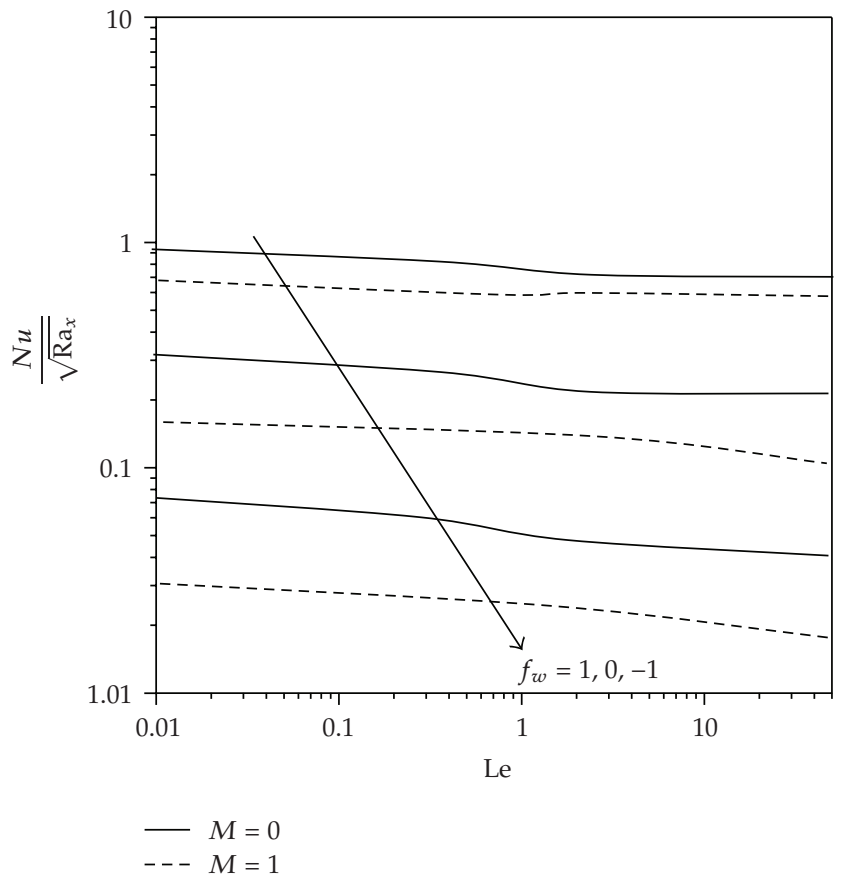

Figure 7: The effect of Lewis number on the Nusselt number $(N=1, \tau=1$, and $\Gamma=1)$ with and without magnetic field effect, for suction, injection, and impermeable wall cases.

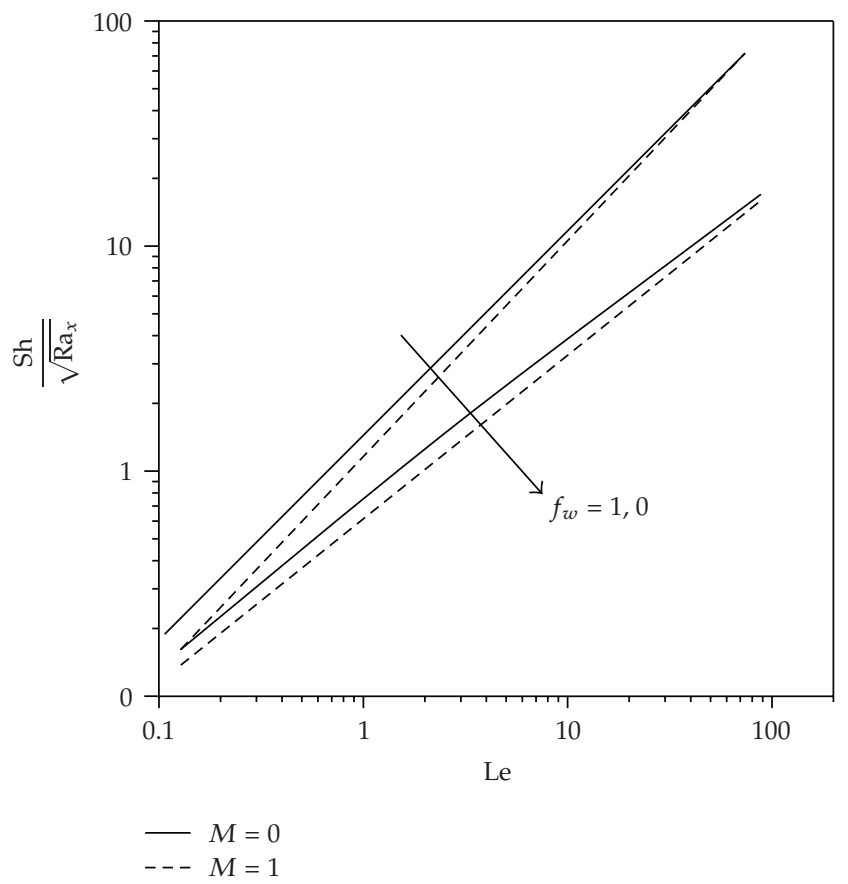

Figure 8: The effect of Lewis number on the Sherwood number $(N=1, \tau=1$, and $\Gamma=1)$ with and without magnetic field effect, for suction, and impermeable wall cases. 


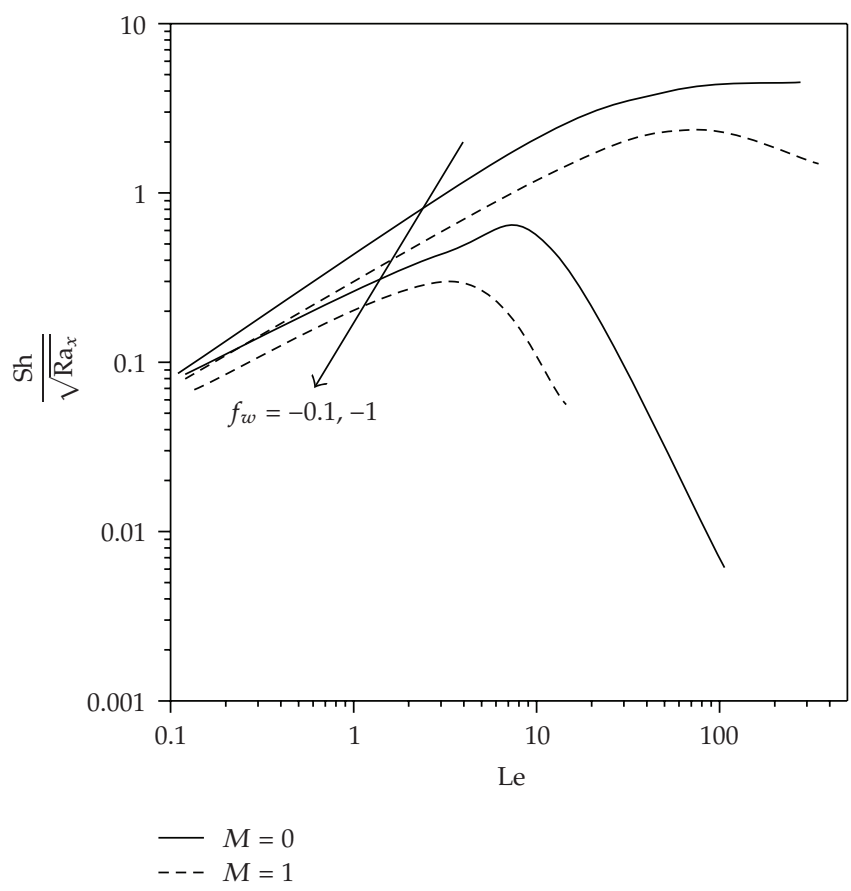

Figure 9: The effect of Lewis number on the Sherwood number $(N=1, \tau=1$, and $\Gamma=1)$ with, and without magnetic field effect, for injection, case.

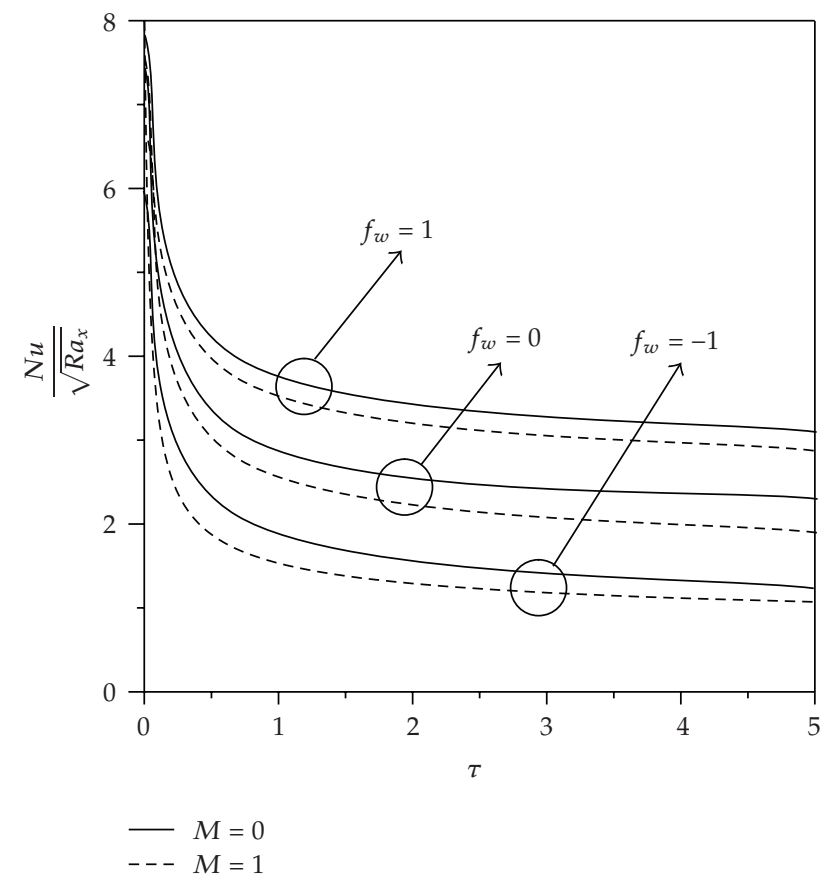

Figure 10: The effect of mass flux parameter on the Nusselt number time evolution with and without magnetic field effect, for suction, injection, and impermeable wall cases $(N=1, \mathrm{Le}=2$, and $\Gamma=1)$. 


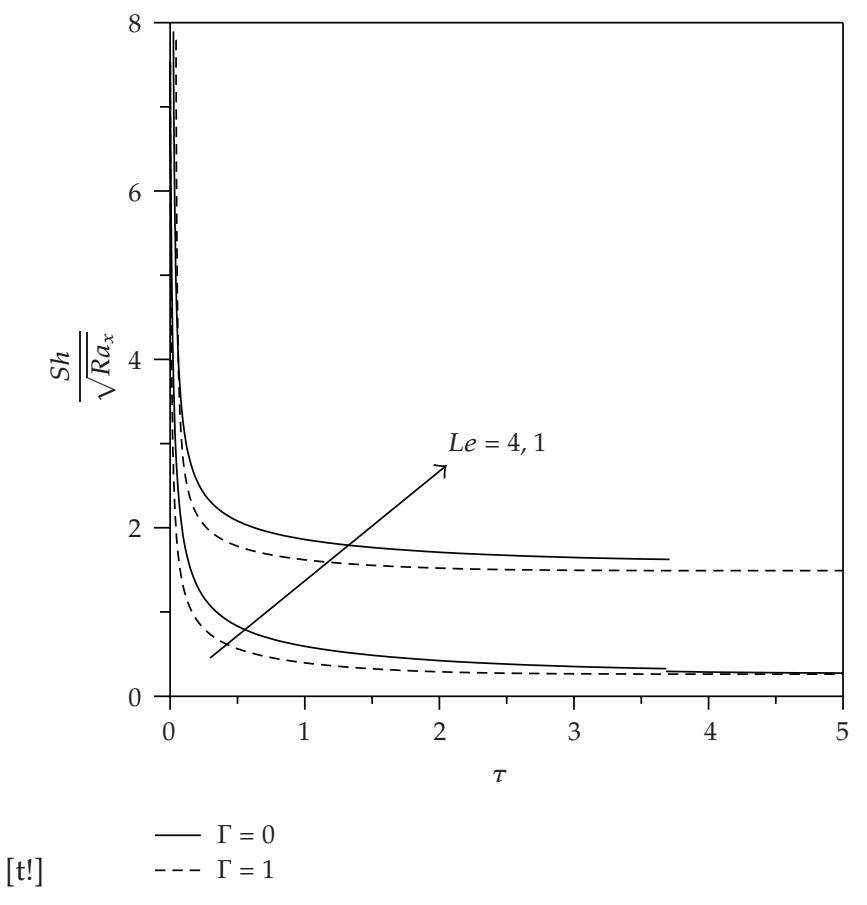

Figure 11: The effect of Lewis number on the Sherwood number time evolution in the Darcy and non-Darcy flow regions, for suction, injection, and impermeable wall cases $(N=1$, Le $=2$, and $M=1)$.

Figures 7 and 8 show the effect of Lewis number on the heat and mass transfer coefficients with and without magnetic field effect and for suction, injection and impermeable wall cases, respectively. It can be seen that the Nusselt number decreases for all $f_{w}$ while the Sherwood number increases for $f_{w} \geq 0$. For the impermeable wall, the Sherwood number increases continually with and without the effects of the magnetic field effects. In the suction case, the magnetic field effect is observed to diminish at large number of Le.

The effect of Lewis number on the local Sherwood number with and without the magnetic field effect in the case of fluid injection into the porous medium is plotted in Figure 9. It can be seen that the Sherwood number increases with Le up to a certain maximum value and then decreases with further increase in Le. The maximum value depends on the buoyancy ratio and the magnetic parameter.

Figure 10 shows the effect of mass flux parameter on the Nusselt number transient behavior with and without magnetic field presence. The heat transfer coefficient has large values at early stages of the double-diffusive process, and then it decreases dramatically with time untill the steady state is reached. Since the presence of the magnetic field effect $(M \neq 0)$ plays the role of stiffening the flow field, is observed that Nusselt number attain a steady state at an earlier time as compared to those in the absence of the magnetic field effect $(M=0)$.

The time evolution of the mass transfer coefficient in the Darcy and non-Darcy flow regions at different Lewis number is illustrated in Figure 11. The mass transfer coefficient has 
large values at early stages of the double-diffusive process, and then it decreases dramatically to reach its steady state values, then it decreases dramatically to reach its steady state value. The non-Darcy effects are predominant at early stages of times.

\title{
4. Conclusions
}

The effects of magnetic field and lateral mass flux on transient MHD double-diffusive of an electrically conducting fluid by free convection over a flat plate embedded in Darcy and nonDarcy porous medium are numerically investigated. It was found that the presence magnetic field lowers both the Nusselt and Sherwood numbers in Darcy as well as Forchheimer flow regimes. Increasing the buoyancy ratio $N$ or the fluid suction increases both Nusselt number and Sherwood numbers. The inertial effects reduce the heat and mass transfer coefficients. The Sherwood number Sh is increased with Lewis number up to a limit, after which it decreases, with further increase in Le. This limit depends on the buoyancy ratio and the inertial and the magnetic parameter. Both the heat and the mass transfer rates tend to decrease as time elapses.

\section{Nomenclature}

\author{
A: Real constant \\ Bo: magnetic induction \\ C: concentration \\ $C_{p}$ : specific heat of the fluid at constant pressure \\ $D$ : concentration molecular diffusion \\ F: inertial coefficient \\ $f$ : dimensionless, reduced stream function \\ $f_{w}$ : suction parameter \\ $g$ : gravitational acceleration \\ Ha: Hartmann number \\ $K$ : permeability of the porous medium \\ $k_{e}$ : effective thermal conductivity of the porous medium \\ Le: Lewis number \\ M: magnetic parameter $\left(\mathrm{Ha}^{2}\right)$ \\ $N$ : buoyancy ratio parameter \\ $\mathrm{Nu}$ : Nusselt number \\ $\mathrm{Ra}_{x}$ : modified Rayleigh number \\ Sh: Sherwood number \\ $T$ : temperature \\ $t$ : time \\ $T_{\infty}$ : free stream temperature \\ $T_{w}$ : wall temperature \\ $u$ : volumetrically averaged axial velocity \\ $v$ : volumetrically averaged lateral velocity \\ $V_{w}$ : suction velocity \\ $x, y$ : coordinates along and normal to the plate, respectively.
}




\section{Greek Letters}

$\alpha_{e}$ : effective thermal diffusivity

$\beta_{C}$ : thermal expansion coefficient

$\beta_{T}$ : concentration expansion coefficient

$\eta$ : similarity parameter

$\Gamma$ : dimensionless inertial parameter

$\theta$ : dimensionless temperature variable

$\phi$ : dimensionless concentration variable

$v$ : effective kinematic viscosity of the fluid

$\sigma$ : electrical conductivity of the fluid

$\rho$ : fluid density

$\tau$ : dimensionless time

$\psi$ : dimensional stream function.

\section{Subscripts}

w: wall

$\infty$ : outer edge of the boundary layer.

\section{References}

[1] P. Singh and Queeny, "Free convection heat and mass transfer along a vertical surface in a porous medium," Acta Mechanica, vol. 123, no. 1-4, pp. 69-73, 1997.

[2] A. Nakayama and M. A. Hossain, "An integral treatment for combined heat and mass transfer by natural convection in a porous medium," International Journal of Heat and Mass Transfer, vol. 38, no. 4, pp. 761-765, 1995.

[3] F. C. Lai and F. A. Kulacki, "Coupled heat and mass transfer by natural convection from vertical surfaces in porous media," International Journal of Heat and Mass Transfer, vol. 34, no. 4-5, pp. 1189$1194,1991$.

[4] K. Vafai and C. L. Tien, "Boundary and inertia effect on flow and mass in porous medium," International Journal of Heat and Mass Transfer, vol. 24, pp. 1189-1194, 1982.

[5] S. Whitaker, "The Forchheimer equation: a theoretical development," Transport in Porous Media, vol. 25, no. 1, pp. 27-61, 1996.

[6] D. M. Manole and J. L. Lage, "The inertial effect on the natural convection flow within a fluid-saturated porous medium," International Journal of Heat and Fluid Flow, vol. 14, no. 4, pp. 376-384, 1993.

[7] P. C. Ram, "Recent developments of heat and mass transfer in hydro-magnetic flow," International Journal of Energy Research, vol. 15, no. 9, pp. 691-713, 1991.

[8] A. J. Chamkha, "MHD-free convection from a vertical plate embedded in a thermally stratified porous medium with Hall effects," Applied Mathematical Modelling, vol. 21, no. 10, pp. 603-609, 1997.

[9] A. Bejan and K. R. Khair, "Heat and mass transfer by natural convection in a porous medium," International Journal of Heat and Mass Transfer, vol. 28, no. 5, pp. 909-918, 1985. 


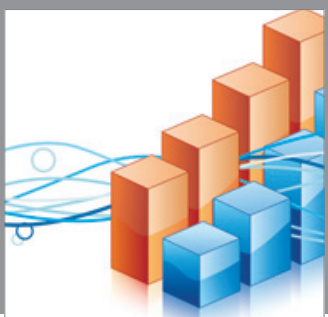

Advances in

Operations Research

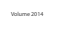

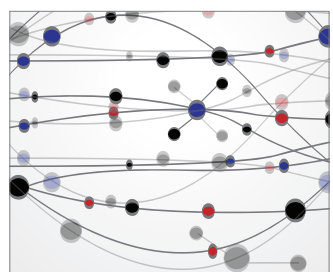

\section{The Scientific} World Journal
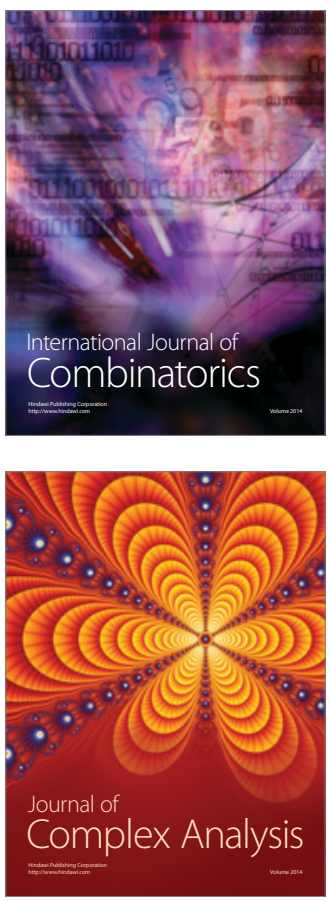

International Journal of

Mathematics and

Mathematical

Sciences
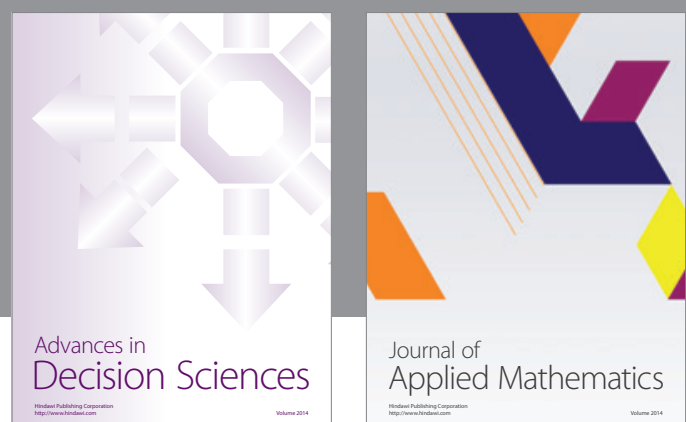

Journal of

Applied Mathematics
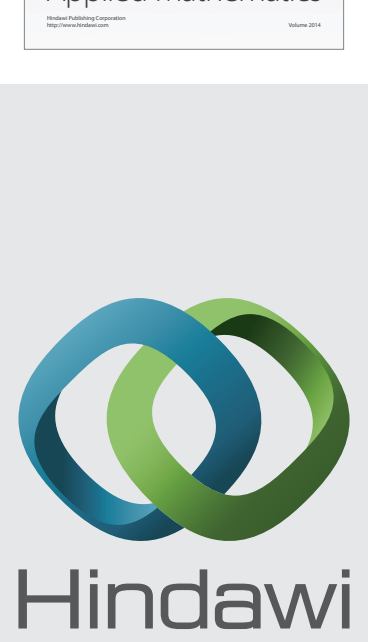

Submit your manuscripts at http://www.hindawi.com
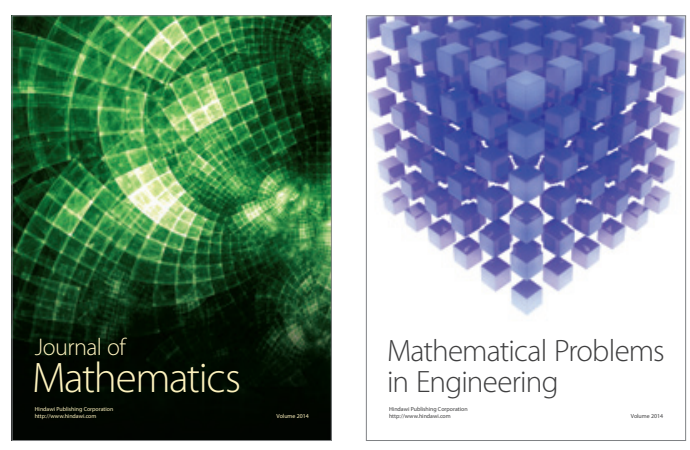

Mathematical Problems in Engineering
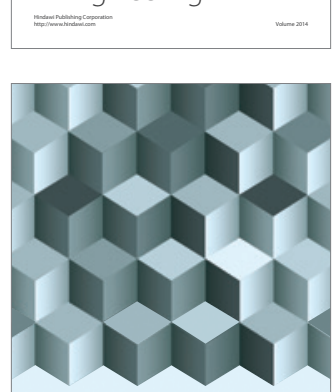

Journal of

Function Spaces
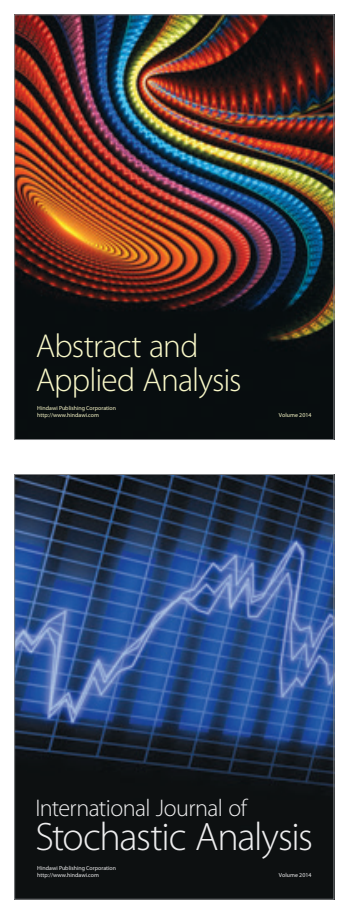

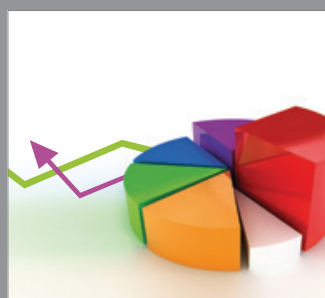

ournal of

Probability and Statistics

Promensencen
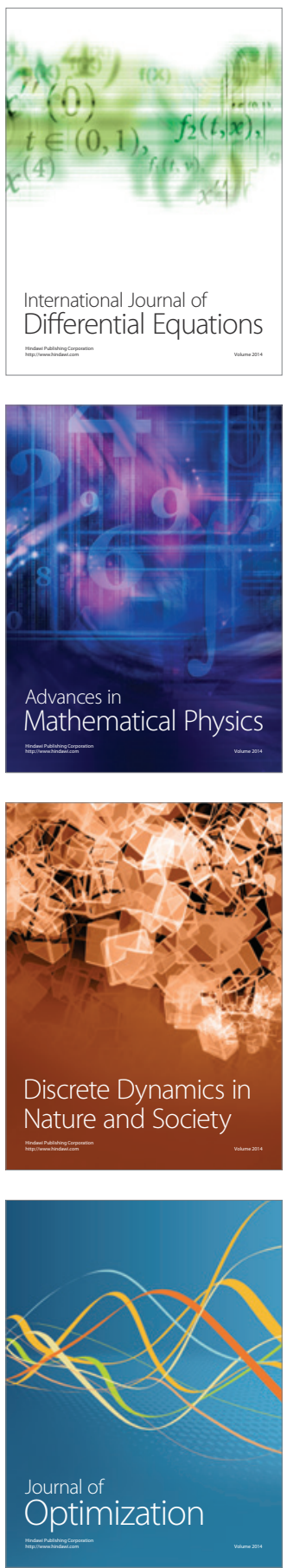